\title{
青蒿素作为有效抗疮疾药物的发现: 一个不同寻常的中国故事
}

\section{昌增益 (1)(3)}

\author{
(1) 北京大学科学史与科学哲学中心, 北京 100871 ; \\ (2) 北京大学生命科学学院, 蛋白质及植物基因研究国家重点实验室, 北京 100871 ; \\ (3) 北京大学跨院系蛋白质科学中心, 北京 100871 \\ E-mail: changzy@pku.edu.cn
}

收稿日期: 2015-03-26; 接受日期: 2015-05-22; 网络版发表日期: 2015-12-15

国家重点基础研究发展计划(批准号: 2012CB721103)和国家自然科学基金(批准号: 21325627)资助

㾏疾(Malaria)是一种由疮原虫(plasmodium)类寄 生虫引起的对人类危害极大的传染性疾病, 在热带 地区特别常见, 仅在非洲地区每年就可导致百万人 的死亡 ${ }^{[1]}$. 中国科学家于 1971 年重新发现中药青蒿 (Artemesia annua $\mathrm{L}$ ) 叶片提取物具有抗症效果, 并于 1972 年鉴定出是其活性成分为“青蒿素”(后被翻译成 英文“arteminisin”), 至 1978 年揭示了该分子的化学 和空间结构, 于 1979 年研发了效力更强同时又能溶 解于水或油的衍生物. 所有这些惊人的科学发现皆 于大约十年的时间内获得, 构成一个独特而又不同 寻常的, 值得给国际国内学术界讲述的中国故事 ${ }^{[2]}$.

尽管青蒿素衍生物, 如二氢青蒿素 (dihydroqinghaosu)、蒿甲醚(artemether)或青蒿琥酯(artesunate) 在杀死疮原虫方面威力极大, 但它们在病人体内被 排出的半衰期都比较短. 而当与其他被排出体内半 衰期比较长的药物联合使用时, 即成为世界上治疗 疮疾的最有效方案. 这种被世界卫生组织推荐的症 疾治疗方法称为青蒿素联合疗法 (artimesinin-combination therapy, ACT). 鉴于青蒿素家族抗疮药物的
研发对人类健康的巨大贡献, 作为这一独特科学发 现故事的主角, 中国科学家屠呦呦研究员被授予了 2015 年诺贝尔生理学或医学奖的一半奖金, 以奖励 “她发现了一种全新的症疾治疗方法”(另外一半奖金 则由爱尔兰科学家威廉·坎贝尔(William Campbell)和 日本科学家大村智(Satoshi Omura)两位学者分享, 以 表彰“他们发现了蜔虫寄生虫疾病的全新治疗方法”).

青蒿素及其衍生物的抗疮效果的发现是一个由 中国政府组织的被称为“523 项目”的集体探究的结果. 之所以如此命名, 是因为该项目启动于 1967 年 5 月 23 日. 该项目旨在为那些被对传统药物(如氯喹)产生 了耐药性的疮原虫感染的病人研发出新的药物, 来 自全国各地大约 60 个单位、超过 500 位的科学家先 后参与了该项目. 该项目的早期研究结果或者从未 发表, 或者仅仅发表在中国出版的学术刊物上, 这些 文章部分是仅用中文发表的，部分是既用中文也用 英文发表的，部分是仅用英文发表. 此类文章的发表 通常是滞后的, 而且经常不列出作者的名字, 甚至也 不列出作者的单位名称, 作者栏里只列为“某某协作

引用格式: 昌增益. 青蒿素作为有效抗疮疾药物的发现: 一个不同寻常的中国故事. 中国科学: 生命科学, 2016, 46: 230-237, doi: 10.1360/N052015-00358

英文版见: Chang Z Y. The discovery of Qinghaosu (artemisinin) as an effective anti-malaria drug: a unique China story. Sci China Life Sci, 2016, 59: 81-88. doi: $10.1007 / \mathrm{s} 11427-015-4988-\mathrm{Z}$ 
组”. 尽管如此, 对公开发表的或没有公开发表的文 献的系统回顾分析冊容置疑地表明, 屠呦呦研究员 在青蒿素发现的各个环节上都发挥了主导作用. 本 文将试图按照这些 20 世纪 70 年代 80 年代发表于中 国出版的学术刊物上的同行评审文章所记录的内容 来讲述这些令人入迷的科学发现. 但有关青蒿素被 最初萃取和分离步骤的描述, 只能参考屠呦呦研究 员于 2009 年出版的《青蒿及青蒿素药物》一书的内 容, 因为在当时发表的同行评审论文(包括中文的和 英文的)中笔者并未见到对这些内容的任何描述.

\section{1 㾏疾是一种灾难性寄生虫疾病}

疮疾(英文“malaria”的本意是“坏的气体”, 因为 这种疾病被认为是从沼泽地的静止水体中产生的瘴 气所致)这种以间歇性一冷一热为主要病征的疾病, 自古记载于所有主要人类文明的历史文献中, 其起 源可能先于人类历史. 对症疾病症的描述出现于几 千年前编撰的中国古典文献中. 尽管在过去的几千 年中人们测试过无数的治疗手段, 但结果多以失败 告终, 成功的案例极其罕见. 疮疾的流行被认为是导 致历史上很多主要政权衰落的原因(包括罗马帝国 等). 据部分医学史学家估计, 地球上曾经有 $1 / 2$ 数量 的人死于疮疾!

\section{1 对㾏疾病因的现代揭示}

19 世纪 70 年代, 法国人巴斯德(Louis Pasteur)和 德国人科赫(Robert Koch)等科学家建立了传染病的 微生物致病学说, 从而使人们在 19 世纪末对症疾有 了更为科学的认识. 通过 19 世纪 80 年代对疮疾致病 细菌的艰辛探索, 人们惊奇地发现疮疾的致病源是 一种单细胞的原生动物类寄生虫, 隶属疮原虫 (Plasmodium) 属, 而非一种细菌. 对这种寄生虫的独 特(通过蚊子叮咬)传染途径的揭示被认为是人类认 识传染病历史中最令人激动的事件之一.

在鉴定疮疾病源方面的最初突破来自法国外科 医生拉韦朗(Alphonse Laveran), 他于 1880 年在非洲 北部国家阿尔及利亚工作时, 从疮疾患者的血液中 观察到这种寄生虫, 立刻认识到其与症疾的关联性 (http: //www.nobelprize.org/nobel_prizes/medicine/ laureates/1907/laveran-lecture.html). 后来曼森(Patrick Manson)和罗斯(Ronald Ross)等人于 19 世纪 90 年代
开展的研究相继在鸟类和人类中揭示, 雌性蚊子是 将疮原虫在个体间传播的媒介(http: //www.nobelprize. org/nobel_prizes/medicine/laureates/1902/ross-lecture. $\mathrm{pdf})^{[3,4]}$. 多年以后, 人们又惊讶地发现, 携带了致病 寄生虫的蚊子叮咬动物(如猴子)后, 疮原虫在转移到 红细胞之前需生活在肝脏细胞中一段时间, 这也揭 开了一个人们长期不解的现象, 即被携带寄生虫的 蚊子叮咬后经过或长或短的时间后才会在被叮咬个 体的血液中观察到疮原虫 ${ }^{[5]}$.

罗斯“因为发现疮原虫是如何进入机体的，从而 为成功寻找到征服这种疾病的方法奠定了基础”而荣 获 1902 年度诺贝尔生理学或医学奖. 拉韦朗则“因为 在原生动物致病方面的工作”而荣获 1907 年度诺贝 尔生理学或医学奖.

\section{2 疮原虫不同寻常的生活史}

疮原虫因需在不同的宿主(如䧳性蚊子和人)体 内完成其生活周期的不同阶段而拥有一种不同寻常 的生活史 ${ }^{[1,6,7]}$. 简单总结如下(图 1): 从雌性蚊子的胃 开始, 疮原虫的每一个卵(oocysts, 即被包裹的受精 卵将分裂形成几千个孢子体(sporozoites). 孢子体再 移至蚊子的唾液腺体内并感染那里的细胞, 然后通 过蚊子的叮咬被注入人体(或其他动物)的血液中. 孢 子体随后将侵染被叮咬者的肝脏细胞而正式开始其 “前红细胞(pre-erythrocyte)”阶段的生活. 在肝脏细胞 中逗留几天后, 孢子体即开始进行多轮的无性繁殖, 产生裂殖子(merozoites), 每一个孢子体可以产生成 千上万的裂殖子. 当一个被感染的肝脏细胞被大量 的裂殖子胀破之后, 裂殖子被释放到血液中, 随后才 会侵染红细胞(被释放出来的裂殖子并不会再次感染 其他肝脏细胞). 在肝脏细胞中的孢子体有一部分会 分化成休眠子(hypnozoites), 后者可以在肝脏中生活 数月到数年, 待苏醒过来后再进行分裂而产生裂殖 子. 这可能是经过药物有效治疗症状消失一段时间 后(如一个月), 疮疾再次复发的主要原因.

在红细胞中, 裂殖子通过无性繁殖产生大量新 的裂殖子, 经过大约 48 或 $72 \mathrm{~h}$ (具体时间依疮原虫种 类不同而不同), 被感染的红细胞被胀破一次, 裂殖 子被释放入血液中. 在红细胞中的裂殖子分裂后, 或 者形成疮原虫的性细胞形式配子体 (gametocytes), 或 者形成非性细胞形式滋养体(trophozoites). 当红细胞 被胀破时, 滋养体将侵染新的红细胞, 这样的侵染过 


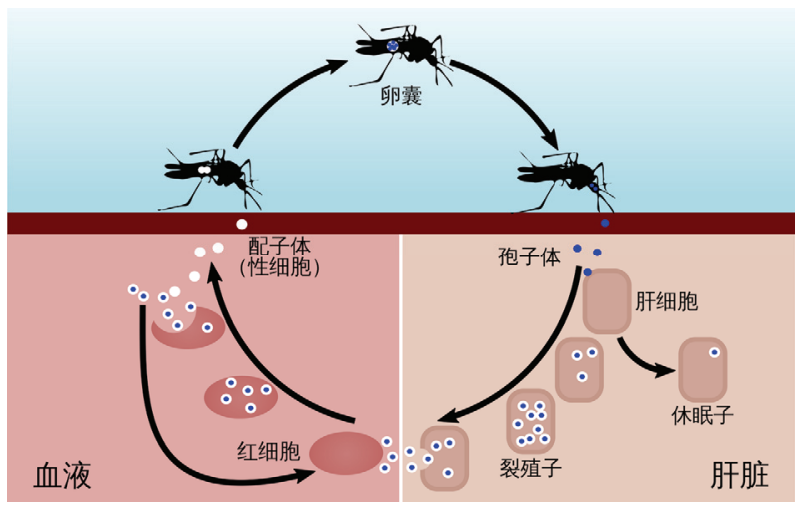

图 1 疮原虫生活史示意图(网络版彩图)

程将周期性地不断重复发生, 导致病人出现周期性 交替发冷和发热的症状, 最后可能因为大量的红细 胞被破坏而出现严重贫血现象. 当患者血液中的配 子体再次进入到叮咬患者的蚊子体内后可以继续发 育成成熟的性细胞, 即雄性配子和雌性配子. 雄性 和雌性配子之间可以通过受精作用而在蚊子的胃 里再次形成卵囊(受精卵)细胞, 开始其新一轮的生活 周期.

疮原虫“神出鬼没”式的复杂生活史给开发有效 的抗疮疫苗和药物带来了较大的困难 ${ }^{[1,6,7]}$, 原因可以 归纳为三点. 其一, 因为疮原虫在其生活史的大部分 时间里, 或者隐藏于肝脏细胞中, 或者隐藏于红细胞 中, 这使得宿主很难对其发起有效的免疫攻击, 也使 人们难以开发出有效的抗疮疾疫苗; 其二, 生活于肝 脏和红细胞中的单倍体形式的症原虫通过有丝分裂 方式进行无性繁殖时, 会发生较多的基因突变, 而生 活于蚊子体内的症原虫进行有性繁殖时双倍体细胞 内还会发生基因重组. 这两种过程都会产生丰富的 遗传多样性, 这使得症原虫细胞表面的蛋白质不断 发生“变脸”, 从而可以有效逃逸宿主的免疫攻击. 其 三，对于那些在肝脏细胞中处于休眠状态的㾏原虫， 药物变得毫无用处, 正如进入了休眠状态的其他类 型的病原体一样 ${ }^{[8,9]}$.

\section{2 在青蒿素之前的抗疮疾治疗药物}

对于人类而言，第一个被用于有效治疗症疾的 药物是一种从金鸡纳树的树皮中提取出来的生物碱 类化合物奎宁 (quinine, 其结构见图 2). 与青蒿的故
事具有几分相似之处的是，在南美洲，金鸡纳树树 皮粉末最初被用于治疗发烧类疾病，后来到 17 世 纪时用于治疗疮疾. 到 19 世纪 20 年代, 抗疮的活性 成分奎宁才被法国的药剂师佩尔蒂埃(Pierre-Joseph Pelletier)和卡文图(Joseph-Bienaimé Caventou)分离出

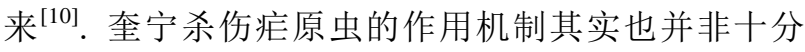
清楚(就像青蒿素一样).

在 20 世纪 20 年代之前, 奎宁是人类对付疮疾的 唯一有效药物, 然而后来对奎宁产生了耐药性的症 原虫株出现了. 1934 年, 德国化学家发现一种具有抗 疮效果的奎宁衍生物氯喹(chloroquine), 它能杀死多 种处于其生活史中不同阶段的疮原虫. 到第二次世 界大战结束时, 药剂师们研发出了更多的抗疮药物. 另外, 被称为滴滴涕(DDT, 即双氯二苯基三氯乙烷) 的杀虫剂也被用于消灭传播疮原虫的蚊子.

一段时间内, 人们普遍认为通过抗症药物治疗 与控制蚊子繁殖两种方式的结合可以帮助人类击败 疮疾这种危害人类几千年的疾病. 这样的乐观性论 调在 1956 年转变成了世界卫生组织的官方计划, 发 起了一场全球性的根除症疾的运动. 但是, 到了 20 世纪 60 年代, 抗氯喹疮原虫致病株及抗 DDT 蚊子的 出现使得疮疾再次肆虐, 60 年代末期, 世界卫生组织 不得不承认根除疮疾暂时是不可能的, 并将其目标 调整为控制该疾病的发生和传播.

\section{3 存在于中药青蒿中的青蒿素被发现为抗 疮活性成分}

为了保持与疮原虫所发生的突变同步, 人类似 乎不得不每过一段时间就研发出新的抗疮药物. 20 世 纪 60 年代, 中国政府启动了规模巨大的“523 项目” 试图找到新的药物，以用于治疗对类似氯喹这样的 传统药物产生耐药性的㾏疾. 该项目的一个方向是 从已有的化合物库中去篮选抗疮药物, 但均以失败 告终. 另外一个方向则是从传统中药的宝库中去寻 找, 正如科学研究过程中所普遍发生的情形, 经过无 数次的失败, 获得了一次的成功. 屠呦呦研究员在第 二种最终获得成功的探寻过程中发挥了重要作用.

\section{1 只有通过合适途径获得的青蒿素抽提物才具 抗疮效应}

虽然在大约 1500 年前的中国医学古籍中就记录 

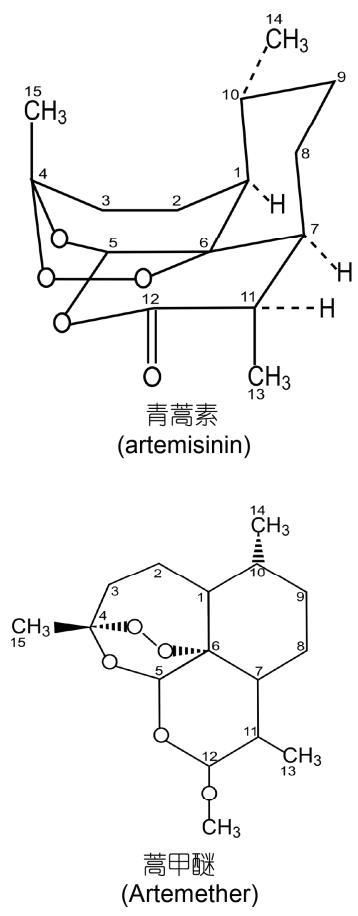

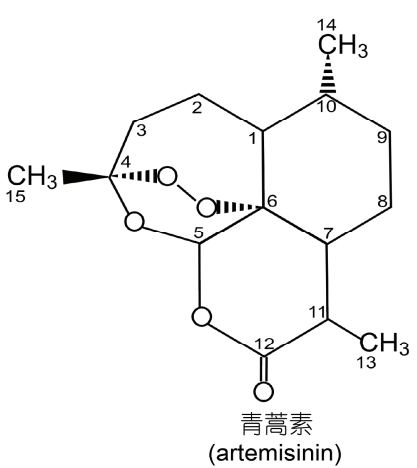<smiles>C[C@@H]1CC[C@H]2[C@@H](C)[C@H](O)O[C@@H]3O[C@]4(C)CC[C@@H]1[C@@]23OO4</smiles>

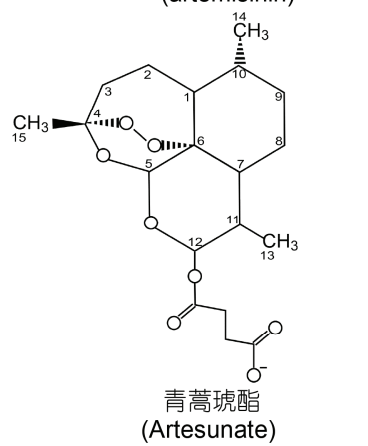

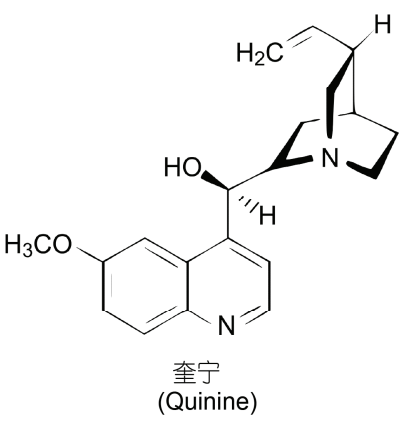

图 2 青蒿素、二氢青蒿素、蒿甲醚、青蒿琥酯和奎宁的化学结构示意图

了青蒿用于治疗疮疾的有效性，但这些早期的实践 仅是基于经验，而非严格的科学实验. 因此，其疗效 难免既不稳定也不显著. 屠呦呦研究员在北京医学 院(实际上在她入学的 1951 年还是北京大学医学院, 1952 年, 国家进行了院系大调整, 北京大学医学院 独立出来, 成为了北京医学院, 后更名为北京医科大 学, 2000年北京医科大学再次并入北京大学成为北京 大学医学部)毕业后, 参加过一个两年的“西医学习中 医”的全职培训班，这为她后来重新发现中药青蒿的 抗㾏效果并继而鉴定纯化出其抗㾏活性成分青蒿素 奠定了良好的基础. 她深刻理解了东晋时期医药学 者葛洪(公元 284 364 年)于 1500 年前所编撰的《肘 后备急方》中有关利用青蒿治疗疮病的一句描述“青 蒿以握, 以水二升渍, 绞取汁, 尽服之”. 她意识到, 与传统中药制备的煎鳌方法不同的是, 在葛洪的描 述中, 青蒿用于抗疮治疗似乎不能高温煎鳌 ${ }^{[11 ~ 14]}$.

屠呦呦研究员所领导的研究小组随即开展了对 青蒿中抗症活性成分的萃取工作. 他们测试了不同 的溶剂(包括水、油、乙醇和甲醚等), 不同的温度, 青 蒿素植株的不同部分, 不同发育阶段的植株等 ${ }^{[15]}$. 结 果表明, 抗疮有效成分主要存在于成熟植物的叶中, 可使用二乙醚或乙醇进行提取, 但操作的温度必须
低于 $60^{\circ} \mathrm{C}^{[2,12,15]}$. 有趣的是, 经过像二乙醚或乙醇提 取的抗疮活性成分非常稳定，即使煮沸 $0.5 \mathrm{~h}$ 后其抗 疮活性也几乎不会受到影响. 为什么最初混合物中 的活性成分经历高温会被破坏, 而溶剂萃取后的活 性成分却能耐高温是一个有待回答的问题.

\section{2 那些表现出毒性但无抗疮活性的成分可以通 过氢氧化钠萃取步骤而被有效去除}

为了减少二乙醚萃取的青蒿成分的毒性，屠呦 呦等人试图进一步将萃取物中的无活性成分与活性 成分进行分离. 为此, 他们在 1971 年发现, 如果用 $2 \%$ 的氢氧化钠处理二乙醚萃取物，则可将其中具有 毒性但却不含抗疮活性的酸性组分有效去除, 而二乙 醚溶剂中剩下的中性组分即为表现出高抗㾏活性、低 毒性的组分 ${ }^{[2,12,15]}$. 这一含有高抗症活性的部分被再 次浓缩，以用于对其中的活性成分进一步分离纯化.

\section{3 利用硅胶柱层析法将青蒿素成功纯化为可结 晶的单一成分}

通过将上述浓缩出的高效低毒的青蒿抽提组分 进一步纯化，屠呦呦等人最终获得了单一的活性成 分. 为此, 他们首先将多聚酰胺加入到浓缩浸膏中, 
摇匀后用 $47 \%$ 的乙醇进行渗滤处理. 然后用二乙醚 将稀醇渗滤液进行另一轮的萃取, 萃取出来的成分 通过硅胶柱层析最后分离, 利用 $10 \%$ 的乙酰酯从硅 胶柱上洗脱下来的部分能够形成针形晶体，并对鼠 疮动物个体表现出完整的抗疮活性, 而使用其他溶 剂, 如石油醚或 $15 \%$ 乙酰酯洗脱下来的组分则不表 现出任何抗疘活性 ${ }^{[15]}$.

\section{4 有关青蒿素抗疮活性的系统研究结果于 1979 年首次公开发表}

尽管青蒿素作为抗㾏活性成分被以晶体形式分 离出来是在 1972 年, 对其结构和药理学的系统研究 也从那时便开始了, 但这些创新结果的发表却被推 迟了多年. 第一篇有关青蒿素的论文发表于 1977 年, 但仅报道了其化学结构, 并未提及任何有关青蒿素 抗疮活性的内容 ${ }^{[16]}$. 两篇 (其实是一篇, 只是分别用 中文和英文发表)系统报道青蒿素抗疮活性的论文发 表于两年以后的 1979 年 ${ }^{[17,18]}$.

尽管在这些早期和后来发表的论文中都没有关 于青蒿素分离纯化方法的描述, 但却详细报道了青 蒿素的化学结构、在治疗动物(包括小鼠、猴子和鸡 等)症疾方面的有效性、动物或人体毒性、临床实验 结果(1973 1978 年, 在中国各地测试过的 2099 位各 类包括氯喹耐药性和脑性疮疾的患者中, 除了大约 10 位因为其他原因死亡外, 其余患者都得到了有效 治疗)、药代动力学(包括利用放射性同位素标记的青 蒿素分析其吸收、器官分布、生物转化和排泄等方面 的特征), 以及一个月后的复发率等 ${ }^{[17,18]}$. 这些研究 结果充分表明, 与氯喹相比, 青蒿素(及其衍生物)是 一类见效快、效率高和毒性低的抗疮新药, 但其缺点 是, 在动物体内被清除的半衰期却比较短, 一个月后 的复发率也较高 ${ }^{[17 \sim 21]}$

值得提及的是，除了能有效治疗脑型疮疾和抗 氯喹疮疾之外, 青蒿素清除血液中疮原虫和发烧症 状所需时间明显短于氯喹等传统抗疮药物. 进一步 分析表明, 青蒿素只对存在于红细胞内的疮原虫表 现出直接的杀伤效用, 而对存在于肝脏细胞中的寄 生虫却无效. 利用电子显微镜进行观察表明, 青蒿素 似乎是直接损害疮原虫细胞中的膜系统, 导致自噬 泡的形成和寄生虫的最终死亡 ${ }^{[15,17,18,22]}$. 青蒿素治疗 后的较高复发率似乎是由于肝脏细胞中处于休眠状 态的疮原虫细胞苏醒所致, 并非是寄生虫基因突变
所致. 考虑到青蒿素的这些优缺点, 世界卫生组织建 议将青蒿素联合治疗方法(the Artemisinin Combination Therapies, 简称为 ACT) 定为疮疾治疗的标准方 法. 这样就大大降低了青蒿素治疗的复发率.

\section{4 青蒿素拥有特殊的多环和不含氮的化学 结构}

有关青蒿素的第一篇论文报道的就是其独特的 化学结构(文章投稿日期为 1976 年 2 月 20 日) ${ }^{[16]}$. 该 文只有一页纸, 发表于《科学通报》中文版上. 作者 一栏里列出的是“青蒿素结构研究协作组”, 也没有 列出任何作者单位的信息。该文对青蒿素的主要物 理化学性质、全面的结构研究结果等进行了总结性描 述. 并提供了青蒿素的相对构型和晶体结构三维电 子密度叠合图。

\section{1 青蒿素被确定为一种含有一个过氧基的新型 倍半萜内酯}

综合的化学和物理分析结果表明，青蒿素是一 种新的倍半萜内酯 (sesquiterpene lactone, 意味着该 分子含有 3 个分子的异戊二烯单位和一个内酯环). 它与当时刚由斯洛伐克学者从青蒿中分离到的另外 一种倍半萜内酯(英文名称为 arteannuin $\mathrm{B}$, 分子式为 $\mathrm{C}_{15} \mathrm{H}_{20} \mathrm{O}_{3}{ }^{[23]}$ ) 的不同之处在于, 青蒿素多了一个独特 的过氧基团 ${ }^{[16,24]}$. 在这些对于青蒿素的分析工作中, 所涉及的方法包括元素分析、高分辨质谱、红外光谱、 ${ }^{1} \mathrm{H}$ 和 ${ }^{13} \mathrm{C}$ 核磁共振(NMR，几乎所有不同种类的氢原 子和所有的 15 个碳原子皆被通过使用 $100 \mathrm{~Hz}$ 的核磁 共振仪得到鉴定)、化学反应分析以及 $\mathrm{X}$ 射线晶体衍 射等。青蒿素的主要性质被鉴定为: 熔点为 $156 \sim 157^{\circ} \mathrm{C}$, 光学活性(即旋光性)为 $+66.3^{\circ}$, 分子式为 $\mathrm{C}_{15} \mathrm{H}_{22} \mathrm{O}_{5}$, 含有一个内酯(即在 $1745 \mathrm{~cm}^{-1}$ 处有一个红 外吸收峰)以及一个过氧基团(即在 $831 \mathrm{~cm}^{-1}, 881 \mathrm{~cm}^{-1}$ 和 $1115 \mathrm{~cm}^{-1}$ 处有红外吸收峰), 但不含碳-碳双 键 ${ }^{[16,24,25]}$. 尽管青蒿素的三维结构图在这些早期的论 文中也被展示和描述 ${ }^{[16 ~ 18]}$, 但关于青蒿素 $\mathrm{X}$ 射线晶 体衍射研究的详细报道在之后才出现.

\section{2 青蒿素的三维空间结构通过 $\mathrm{X}$ 射线衍射方法 测定}

对由中医研究院药物研究所青蒿研究小组提供 
的晶体所开展的详细 X-射线单晶衍射研究, 揭示了 青蒿素分子的三维空间结构, 包括其中的 7 个不对称 碳原子的绝对构型 ${ }^{[26,27]}$ (这其实是同一篇文章的中英 文版本, 内容完全相同, 皆于 1978 年 5 月 9 日投稿). 在此项工作中, X 射线衍射工作是利用一台 PW-1100 四圆衍射仪开展的. 结构是通过符号附加法解析的, 并通过全矩阵最小二乘法进行了修正, 对 1553 个反 射分析获得的 $R$ 因子为 0.085 , 对 1299 个可观察反射 获得的 $R$ 因子为 0.074 . 青蒿素分子中所有不对称碳 原子的绝对构型皆被测定. 结果揭示, 青蒿素分子中 的 15 个碳原子和 5 个氧原子连成了 4 个环, 分别被 命名为 $\mathrm{A}, \mathrm{B}, \mathrm{C}$ 和 $\mathrm{D}$ (图 2). 具体而言, $\mathrm{A}$ 环为一个椅 型环己烷, $B$ 和 $C$ 环皆为饱和的氧杂环, 而 $\mathrm{D}$ 环为一 个 delta-内酯. 有趣的是, 青蒿素的所有 5 个氧原子 皆位于分子的一侧(图 2), 形成一个独特的碳-氧原子 链 $\left(\mathrm{O}-\mathrm{C}_{12}-\mathrm{O}-\mathrm{C}_{5}-\mathrm{O}-\mathrm{C}_{4}-\mathrm{O}-\mathrm{O}-\mathrm{C}_{6}\right)$. 显然, 这个碳-氧链使 得其中的过氧基团对热和光都具有不同寻常的稳定 性. 这一独特的碳氧链究竟是如何使得青蒿素具有 抗疮活性的还有待进一步研究.

\section{5 二氢青蒿素和其他青蒿素衍生物表现出 更强的抗疮效用}

青蒿素的低水溶性和高复发率等弱点促使中国 科学家们试图开发青高素的衍生物, 正如人们在开 发奎宁时所做的那样. 为此, 他们发现利用嗍氢化钠 $\left(\mathrm{NaBH}_{4}\right)$ 处理青蒿素时产生了二氢青蒿素(dihydroqinghaosu 或 dihydro-artemesinin, 结构见图 2). 这就 使得青蒿素分子中唯一的羰基被还原成了羟基, 多 种化学基团后来被共价连接到这个羟基上(也只能连 接到这个羟基上), 产生了大量青蒿素衍生物, 包括 醚类、酯类和碳酸盐类 ${ }^{[28,29]}$. 当用于治疗被具有氯喹 抗性的鼠症原虫感染的小鼠时, 人们发现二氢青蒿 素和多种二氢青蒿素的衍生都表现出了比青蒿素自 身更强的抗㾏活性 ${ }^{[30 \sim 33]}$. 这些研究同时表明, 只有 当青蒿素分子中的过氧基被保存时, 衍生物才具有 抗疮活性. 换句话说, 过氧基为这类分子发挥抗㾏活 性的关键所在 ${ }^{[30,31]}$. 这些合成和测试过的青蒿素衍 生物中包括了后来开发为常用抗疮药物的蒿甲醚 (artemether, 结构见图 2; 即一个甲基连接于羟基上, 因此具有很高的脂溶性 ${ }^{[29 \sim 33]}$. 另外一种后来开发为
常用抗疮药物、具有很高的水溶性和抗㾏活性的衍生 物是青蒿琥酯(artesunate, 结构见图 2, 即一个琥珀酸 分子以其单酯的形式连接在羟基上, 因此而具有很 高的水溶性 ${ }^{[29,31,33]}$.

\section{6 世界对青高素抗疮效果的认可}

尽管青蒿素的化学结构到 1977 年才被首次发 表 ${ }^{[16]}$, 其抗症效果于两年后通过两篇论文的发表才 对外界公布 ${ }^{[17,18]}$, 但这迅速引起了广泛关注. 世界 卫生组织与联合国开发计划署以及世界银行一起, 于 1981 年 10 月 6 10 日在北京组织了一个特别的 会议, 即“第四次关于疮疾化学疗法的科学研讨 会”,[11,12,14,34,35]. 青蒿素的显著抗疮活性和不同寻常的 不含氮的化学结构全部是第一次被揭示, 而且完全 是由中国科学家在动荡的文化大革命时期完成的, 这个发现让世界惊叹(甚至可以说是震惊不已) $)^{[1113,35]}$. 这一独特结构的正确性被进一步通过完全人工的方 法合成得到了确认. 这一艰难而杰出的合成工作是 由中国科学家 ${ }^{[36]}$ 和瑞典科学家 ${ }^{[37]}$ 几乎同时完成的. 然而此人工合成方法因其复杂性及费用过高等问题 至今也未能被应用于青蒿素药物的大规模生产. 也 就是说, 我们仍旧必须依赖一个多步骤的生物合 成途径来制造青蒿素, 以用于抗疮青蒿素类药物的 生产.

当中国科学家开发了蒿甲醚(用于口服)和青蒿 琥酯(用于注射)作为常用抗疮药物时 ${ }^{[30 \sim 32]}$, 它们在国 外几乎被完全忽视. 一方面, 症原虫对已有药物(如 奎宁、氯喹和甲氟奎等)的耐药问题不断突现, 而另一 方面, 不断积累的实验证据支持青蒿素家族药物的 快速性、可靠性和安全性. 这些事实迫使国外的科学 家改变了态度, 将目光转向了完全由中国科学家发 现和开发的抗疮药物 ${ }^{[113]}$. 第一份在国外报道的有 关青蒿素在抗㾏方面优于传统药物的权威学术刊物 是柳叶刀 (Lancet $)^{[38]}$. 有关从青蒿中提取出来的青蒿 素具有出色抗㾏效果的事实也很快被美国科学家所 独立证实 ${ }^{[39]}$. 后来, 青蒿素及其衍生物(包括二氢 青蒿素、蒿甲醚、青蒿琥酯等), 成为世界各地医生

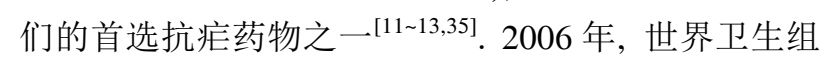
织正式宣布青蒿素复方疗法为世界各地的标准抗症 处方 ${ }^{[40]}$. 


\section{7 总结和展望}

中药青蒿的抗疮效果的发现是一个发生在中国 的艰难曲折的故事. 中国科学家最初从青蒿中提取 出抗疮活性混合物, 进一步艰难地从中分离出单一 活性成分青蒿素, 并测定了其独特的化学结构, 从而 在此基础上制备了大量衍生物, 发现其中小部分表 现出了比青蒿素本身更好的抗症效果等. 发现青蒿 素及其衍生物的抗㾏效果优于传统的抗疮药物, 特 别是它们能有效对付那些对传统药物产生了耐药性 的疮原虫, 这一系列的研究成果代表了中国科学对 促进人类健康做出的重大贡献. 期望能从已有几千
年历史的中国传统医药宝库里挖掘出更多的宝藏, 以服务于人类的健康.

正像所有的科学研究工作一样, 对于青蒿素及 其衍生物的作用机制, 仍然存在大量未解之谜. 比如, 它们是如何杀死㾏原虫的呢 ${ }^{[22]}$ ? 为什么它们只能杀 死存活在红细胞中的而不能杀死存活于肝脏中的症 原虫呢? 为什么青蒿需要青蒿素呢? 耗费大量的能 量, 经过一个或多个步骤的代谢途径来合成青蒿素 对植物有什么益处呢? 是否有其他植物也能合成青 蒿素? 其目的又是什么? 如果有其他植物也能合成 青蒿素，它们的功能应该是类似的. 类似这些问题需 要进一步探究.

\section{参考文献}

1 Miller L H, Ackerman H C, Su X Z, Wellems T E. Malaria biology and disease pathogenesis: insights for new treatments. Nat Med, 2013, 19: $156-167$

2 Su X Z, Miller L H. The discovery of artemisinin and the Nobel Prize in Physiology or Medicine. Sci China Life Sci, 2015, 58: 1175-1179

3 Ross R, Smyth M. On Some peculiar pigmented cells found in two mosquitos fed on malarial blood. British Med J, 1897, 2: 1786-1788

4 Ross R. The role of the mosquito in the evolution of the malaria parasite. Lancet, 1898, ii: 489-498

5 Shortt H E, Garham P C C. Pre-erythocytic stages in mammalian malaria parasites. Nature, 1948, 161: 126-126

6 Thera M A, Plowe C V. Vaccines for Malaria: How close are we? Annu Rev Med, 2012, 63: 345-357

7 Winzeler E A. Malaria research in the post-genomic era. Nature, 2008, 455: 751-756

8 Wang Y, Ezemaduka A N, Tang Y, et al. Understanding the mechanism of the dormant dauer formation of C. elegans: from genetics to biochemistry. IUBMB Life, 2009, 61: 607-612

9 Liu J F, Fu X M, Chang Z. Hypoionic shock treatment enables aminoglycosides antibiotics to eradicate bacterial persisters. Sci Rep, 2015 5: 14247, doi: 10: 1038/srep14247

10 Kaufman T S, Rúveda E A. The quest for quinine: those who won the battles and those who won the war. Angew Chem Int Ed Engl, 2005, 44: $854-885$

11 Klayman D L. Qinghaosu(Artemisinin): an antimalarial drug from China. Science, 1985, 228: 1049-1055

12 Miller L H, Su X Z. Artemisinin: discovery from the Chinese Herbal Garden. Cell, 2011, 146: 855-858

13 White N J. Qinghaosu (Artemisinin): the price of success. Science, 2008, 320: 330-334

14 Tu Y Y. The discovery of artemisinin(qinghaosu) and gifts from Chinese Medicine. Nat Med, 2011, 17: 1217-1220

15 屠呦呦. 青蒿及青蒿素类药物. 北京: 化学工业出版社, 2009

16 青蒿素结构研究协作组. 一种新型的倍半萜内酯一一青蒿素. 科学通报, 1977, 22: 142

17 青蒿研究协作组. 抗疮新药青蒿素的研究. 中国药学杂志, 1979, 14: 49-53

18 Qinghaosu Antimalaria Coordinating Research Group. Anti-malaria studies on Qinghaosu. Chin Med J(Engl), 1979b, 92: 811-816

19 China Cooperative Research Group on Qinghaosu and its Derivatives as Antimalarials. Metabolism and pharmacokinetics of qinghaosu and its derivatives. J Tradit Chin Med, 1982, 2(1): 25-30.

20 China Cooperative Research Group on Qinghaosu and Its Derivatives as Antimalarials, Studies on the toxicity of qinghaosu and its derivatives. J Tradit Chin Med,1982, 2(1): 31-38

21 Li G Q, Guo X B, Jin R, et al. Clinical studies on treatment of cerebral malaria with qinghaosu and its derivatives. J Tradit Chin Med, 
1982, 2(2): 125-130.

22 Zhou B. Artemisinin (Qinghaosu): a mesmerizing drug that still puzzles. Sci China Life Sci, 2015, 58: 1151-1153

23 Jeremic D, Jokic A, Behbud A, Stefanovic M. A new type of sesqiterpene lactone isolated from Artemisia annua L. arteannuin B. Tetrahedron Lett, 1973, 14: 3039-3042

24 China Cooperative Research Group on Qinghaosu and its Derivatives as Antimalarials. Chemical Studies on Qinghaosu (Artemisinine). J Tradit Chin Med, 1982, 2: 3-8

25 刘静明, 倪慕云, 校菊芬, 等. 青蒿素(Arteannuin)的结构和反应. 化学学报, 1979, 37: 129-143

26 中国科学院生物物理研究所青蒿素协作组. 青蒿素的晶体结构及其绝对构型. 中国科学, 1979, 22: 1114-1128

27 Qinghaosu Research Group of the Institute of Biophysics of the Chinese Academy of Sciences. Crystal structure and absolute configuration of qinghaosu. Scientia Sinica, 1980, 23: 380-396

28 李英, 虞佩琳, 陈一心, 等. 青蒿素衍生物的合成. 科学通报, 1979, 24: 667-669

29 China Cooperative Research Group on Qinghaosu and its Derivatives as Antimalarials. The chemistry and synthesis of Qinghaosu derivatives. J Tradit Chin Med, 1982, 2: 9-16

30 顾浩明，吕宝芬，矍志祥. 青蒿素衍生物对伯氏疮原虫抗氯喹株的抗疮活性. 中国药理学报, 1980, 1: 48-50

31 China Cooperative Research Group on Qinghaosu and its Derivatives as Antimalarials. Antimalarial efficacy and mode of action of qinghaosu and its derivatives in experimental models. J Tradit Chin Med, 1982, 2: 17-24

32 顾浩明, 刘明章, 吕宝芬, 等. 蒿甲醚在动物的抗疮作用和毒性. 中国药理学报, 1981, 2: 138-144

33 China Cooperative Research Group on Qinghaosu and Its Derivatives as Antimalarials. Clinical studies on the treatment of malaria with qinghaosu and its derivatives. J Tradit Chin Med,1982, 2: 45-50

34 JTCM News. The $4^{\text {th }}$ Meeting of the SWG-CHEMAL to discuss the development of China's new antimalarial agent qinghaosu and its derivatives. J Tradit Chin Med, 1981, 1: 112

35 Bruce-Chwatt L J. Qinghaosu: a new antimalarial. Brit Med J, 1982, 284: 767-768

36 许杏祥, 朱杰, 黄大中, 等. 青蒿素及其一类物结构和合成的研究 X. 从青蒿酸立体控制合成青蒿素和脱氧青蒿素. 化学学报, 1983, 41: 574-576

37 Schmid G, Hofheinz W. Total synthesis of Qinghaosu. J Am Chem Soc, 1983, 105: 624-625

38 Jiang J B, Li G Q, Guo X B, et al. Antimalarial activity of mefloquine and qinghaosu. Lancet, 1982, 2: 285-288

39 Klayman D L, Lin A J, Acton N, et al. Isolation of artemisinin (Qinghaosu) from Artemisia annua growing in the United States. J Nat Prod, 1984, 47: 715-717

40 Eastman R T, Fidock D A. Artemisinin-based combination therapies: a vital tool in efforts to eliminate malaria. Nat Rev Microbiol, 2009, 7: $864-874$ 